\title{
Allergisch auf Sport
}

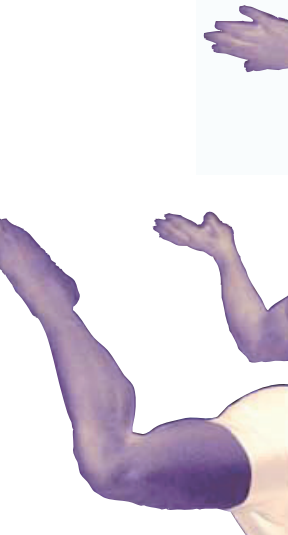

„Schwimmen auf vollem Bauch ist gefährlich!“ Das weiß jedes

Kind. Aber dass ein Grund dafür eine nahrungsmittelabhängige anstrengungsinduzierte Allergie sein kann, wissen auch die wenigsten Erwachsenen.

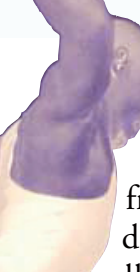

ömische Allergologen befragten 54 Patienten, die auf Sport mit allergischen Symptomen reagiert hatten. Dieser Erhebung nach kam es durchschnittlich 30 bis 50 Minuten post cenam bei Aufnahme körperli-
cher Betätigung zu folgenden Symptomen: Pruritus (94\%), Urtikaria (85\%), Quincke-Ödem (83\%), Flush (74\%), Dyspnoe (70\%), Diarrhoe, Koliken oder Erbrechen (35\%), Blutdruckabfall mit Kollaps (32\%), Bronchospastik und Husten (30\%). Die Episoden traten einmalig oder wiederholt auf. Bis zum Auftreten der klinischen Symptomatik verstrichen 10 bis 50 Minuten. Acht Patienten erlitten die Symptome erst in der Erholungsphase. Fünf Patienten hatten vor dem Ereignis ASS eingenommen. Die Patienten sollten sich erinnern, was sie im Einzelnen bis 24 Stunden vor dem Ereignis zu sich genommen hatten, welche Nahrungsmittel symptomlos vertragen und welche möglicherweise Krankheitserscheinungen auslösten.

Die römischen Wissenschaftler führten bei jedem Patienten eine Kombination verschiedener Allergietests durch: Pricktest mit nativem Material und kommerziellen Testlösungen, RAST- und CAP-Test. Etwa 30\% der Patienten boten eine saisonale Symptomatik. Am häufigsten fanden sich Sensibilisierungen auf Pollen, gefolgt von Hausstaubmilben. Nahezu alle Patienten reagierten positiv auf ein oder mehrere Nahrungsmittel-Allergene, v.a. Petersilie, Apfel, Soja, Erdnuss, Erbsen, Getreidearten. Die von den Patienten als krankheitsauslösend verdächtigten Nahrungsmittel reagierten im Test regelmäßig positiv.
Da die Patienten nach gleichartiger Nahrungsaufnahme und vergleichbarer körperlicher Belastung ihre klinische Symptomatik nicht regelmäßig reproduzieren konnten, vermuteten die Autoren anderweitige Einflussfaktoren: In erster Linie die Einnahme von nicht steroidalen Antiphlogistika, welche die Nahrungsmittelresorption aus dem $\mathrm{Ma}$ gen-Darm-Trakt erhöhen, dann auch Menstruation, Stress und Kälte.

Wurden alle im Test positiv reagierenden Nahrungsmittel eliminiert, trat bei z.T. jahrelangen Verlaufskontrollen keine anstrengungsabhängige Symptomatik mehr auf. Als praktisch erwies sich die Erkenntnis, dass trotz beliebig zusammengesetzter Nahrung ein vierstündiges Fasten vor körperlicher Belastung vor anstrengungsinduzierter Anaphylaxie schützt.

\section{Fazit}

Die anstrengungsinduzierte Anaphylaxie ist als eine zum Teil lebensgefährliche Sonderform einer Nahrungsmittelallergie aufzufassen. Zur Diagnostik bedarf es einer ausgefeilten Anamnese bezüglich der klinischen Symptomatik, krankheitsauslösender Nahrungsmittel sowie einer umfassenden Allergiediagnostik. Differenzialdiagnotisch ist auf eine cholinergische Urtikaria zu achten. Dr. D. Bruchhausen, Wuppertal

Romano A et al. Food-dependent exercise-induced anaphylaxis: Clinical and laboratory findings in 54 subjects. Int Arch Allergy Immunol 2001; 125: 264-72

\section{Dicken Kindern fällt das Atmen schwer}

Studienergebnisse aus London bestätigen einen Zusammenhang zwischen Adipositas und Asthma bei Grundschülern.

$M$ ehrere Studien haben bereits eine positive Korrelation zwischen Asthma bronchiale und Übergewicht bei Erwachsenen gezeigt. Britische Wissenschaftler prüften diesen Zusammenhang jetzt bei 14.908 vierbis elfährigen Kindern. Dazu bestimmten sie Body-Mass-Index (BMI) und die Summe der Hautfalten subskapulär und über dem Trizeps. Altersentsprechende Normwerte für Großbritannien lagen vor. Zudem befragten sie die Mütter bezüglich der AsthmaSymptome ihrer Kinder.

Unabhängig von Rasse, Geschlecht und sozialer Herkunft fand sich ein statistisch signifikantes $\mathrm{Zu}$ sammentreffen von Asthma bronchiale und Übergewicht, definiert durch den BMI. Zwischen Hautfaltendicke und Asthma bronchiale fand sich ein solcher Zusammenhang nicht. Außerdem gab es Hinweise einer stärkeren Assoziation bei Mädchen.
Offen bleibt die Frage, ob Übergewicht ein ätiologischer Faktor für Asthma bronchiale ist, wofür sich in der Literatur Anhalt findet, oder ob dieses Folge desselben auf Grund einer krankheitsbedingten, überwiegend sitzenden Lebensweise ist.

\section{Fazit}

Für die Praxis bleibt festzuhalten, dass kindliches Asthma bronchiale überzufällig häufig kombiniert mit Übergewicht vorkommt. In diesen Fällen ist in besonderer Weise auf eine konsequente antiasthmatische Therapie zusammen mit Adipositas reduzierenden Maßnahmen zu achten.

Dr. D. Bruchhausen, Wuppertal

Figueroa-Muñoz Jl et al. Association between obesity and asthma in 4-11 year old children in the UK. Thorax 2001; 56: $133-7$ 